\title{
Effect of Changing Table Tennis Ball Material from Celluloid to Plastic on the Post-Collision Ball Trajectory
}

\author{
by \\ Yuki Inaba , Sho Tamaki², Haruhiko Ikebukuro ${ }^{3}$, Koshi Yamada ${ }^{4}$, Hiroki Ozaki , \\ Kazuto Yoshida ${ }^{6,7}$
}

\begin{abstract}
The official material used in table tennis balls was changed from celluloid to plastic, a material free of celluloid, in 2014. The purpose of this study was to understand the differences and similarities in the two types of ball materials by comparing their behavior upon collision with a table. The behavior of the balls before and after collision with a table, at various initial speeds ranging from 15 to $115 \mathrm{~km} / \mathrm{h}$, was captured using high-speed cameras. Velocities and spin rates before collision and velocities after collision were computed to calculate the coefficients of restitution and friction. Based on the computed variables, the post-collision trajectories of both balls were calculated by integrating the equation of motion of the ball for simulated service, smash and drive conditions with respect to time. The coefficients of restitution were higher for the plastic balls than the celluloid ones when the initial vertical velocities were higher. The coefficients of friction were higher for plastic balls when the initial horizontal contact point velocities were slower. Because of the differences in the material characteristics, the plastic ball trajectories of services with backspin and drives with great topspin were expected to be different from those of celluloid balls. Since the extent of differences between the two ball types varied depending on the initial conditions, testing at various initial conditions was suggested for comparing and understanding the characteristics of the balls.
\end{abstract}

Key words: table tennis, coefficient of restitution, coefficient of friction.

\section{Introduction}

As can be seen from watching table tennis games of elite players, they are capable of extremely fast rallies. The translational velocity of a ball right after it is hit by a racket can reach speeds up to $100 \mathrm{~km} / \mathrm{h}$ (Xie et al., 2002). In addition, players often apply various types of spin to the ball, which greatly influences the behavior of the ball after it is hit by a racket or bounces off a table (Tiefenbacher and Durey, 1994). Since the time to react to the ball is limited because of the fast rallies, it is assumed that players anticipate the approximate amount and direction of the spin applied to the ball, velocity of the ball and the resulting trajectory of the ball that is returned by the opponent player. If the players do not accurately predict the ball spins and simply swing the racket without adding any counter-spin movements, then the balls do not fly in the

1 - Department of Sports Science, Japan Institute of Sports Sciences, Japan.

2 - Department of Sports and Health, Meio University, Japan.

3 - Japan Table Tennis Association, Japan.

4 - Table Tennis Friendship Club, Japan.

5 - Singapore Sports Institute, Sport Singapore, Singapore.

6 - Faculty of Education, Shizuoka University, Japan.

7 - Sports Science and Medical Committee, International Table Tennis Federation, Lausanne, Switzerland. 
desired direction. The fact that players can maintain such fast rallies during the game, despite the various spin on the ball, is obvious evidence that they anticipate or predict the spin applied to the ball, in addition to reacting to it. Therefore, it is believed that players accumulate information regarding how the behavior of the ball in various circumstances would be through long time practice and experience.

Previously accumulated information regarding the behavior of table tennis balls primarily pertained to celluloid balls, which have been used appreciably since their introduction in 1900

(http://www.ittf.com/media/History/Timeline_His tory.pdf). However, the ball material was changed from celluloid to "plastic" by the International Table Tennis Federation (ITTF) for most of the international events held after July, 2014. Here, plastic balls were defined as "balls which do not contain celluloid" (Küneth, 2015). This new material was introduced as celluloid balls were believed to be expensive and flammable, thereby creating a fire hazard during transportation. Even though the ITTF set the specifications for the newly approved official plastic balls similar to those of the previously approved celluloid balls, it was expected that there would be some differences between them. As mentioned above, since players have accumulated information regarding the behavior of celluloid balls, considering that this behavior changes due to the new material, the players' prediction scales need to be adjusted accordingly.

Meyer and Tiefenbacher (2012) reported that seamless plastic balls showed greater deceleration in the horizontal direction and higher rebound in the vertical direction than celluloid balls. Moreover, they reported that players recognized certain changes between the behavior of the celluloid and plastic balls. Thus, it is expected that some differences exist between old and new balls. However, since the behavior of the balls differs greatly depending on the conditions how they are hit by a racket or how they collide with the table, a detailed analysis under a controlled initial condition is needed to understand the differences and similarities between them. Therefore, the main purpose of this study was to compare the characteristics of the plastic balls with those of the celluloid balls and determine potential differences under various initial ball impact conditions. In addition, since the criteria for approved plastic balls were modified to be more stringent in January, 2016, it was expected that plastic balls comprised of the renewed material would be sold, and companies producing plastic balls would continue to improve the quality of the balls. Since the official game ball varies depending on the event, players must adjust to different balls for each event. Thus, the secondary purpose of this study was to identify effective ways of evaluating the characteristics of the balls.

\section{Material and Methods}

\section{Experimental Set-up}

The characteristics of celluloid and plastic balls were analyzed by quantifying the behavior of the balls before and after collision with the table. Both the celluloid and plastic balls used in this study contained seams, had same quality ranking and were produced by the same company (3-star Premium, Nittaku). Five balls were randomly selected from each type of balls for the study and were tested under the various conditions detailed below. The mean diameter of the celluloid balls was $3.96 \pm 0.00 \mathrm{~cm}$, and that of the plastic balls was $4.00 \pm 0.02 \mathrm{~cm}$. The mean mass of the celluloid balls was $2.742 \pm 0.007 \mathrm{~g}$, and that of the plastic balls was $2.726 \pm 0.008 \mathrm{~g}$. .

The balls were launched from a table tennis ball machine that controls the spin and velocity of the ball using three rotors (Ozaki et al., 2013). By modulating the spin rate of the rotors, five different sets of velocity conditions were designed, ranging from 15 to $115 \mathrm{~km} / \mathrm{h}$. For all velocity conditions, back-spin was applied to the balls. The behavior of the balls before and after collision with the table was recorded using two synchronized high-speed cameras at $1000 \mathrm{~Hz}$ (Phantom V310, Vision Research).

\section{Analysis}

Detection of and computation of position of center of ball

Based on the brightness and configuration of the ball, the position of the ball in each of the two camera's images was detected (Tamaki and Saito, 2015). The three-dimensional positional data of the center of the ball was reconstructed using a three-dimensional direct linear transformation (DLT) method. The reconstructed 
positional data were divided into before-collision and after-collision trajectory groups, then the center of the ball of each group was smoothed individually using a Savitzky Golay filter (Savitzky and Golay, 1964). Then the velocities of the ball immediately before (vertical component: $v_{z_{-} \text {before }}$ and horizontal component: $v_{h_{-} \text {before }}$ and immediately after (vertical component: $v_{z_{-} \text {after }}$ and horizontal component: $\left.v_{h_{-} \text {after }}\right)$ collision with the table were calculated. The spin rate of the ball prior to collision with the table $\left(\omega_{\text {before }}\right)$ was calculated by measuring the time it took to complete a single rotation. Random alphabets and numbers marked on the balls were used to identify the time it took the ball to complete one rotation.

\section{Coefficients of restitution and friction}

The coefficient of restitution $(e)$ was calculated using the following equation:

$$
e=\frac{v_{z_{-} a f t e r}}{v_{z_{-} \text {before }}}
$$

where $v_{z_{-} \text {before }}$ and $v_{z_{-} \text {after }}$ are the vertical components of the velocity of the center of the ball immediately before and after collision with the table. For computing the coefficient of friction $(\mu)$, the equation that expresses the relationship between the state of the ball before and after the bounce (Shibukawa, 1969) was modified:

$$
\mu=\frac{v_{h_{-} \text {before }}-v_{h_{-} \text {after }}}{(1+e) * v_{z_{-} \text {before }}}
$$

where $v_{h_{-} \text {before }}$ and $v_{h_{-} \text {after }}$ are the horizontal components of the velocity of the center of the ball immediately before and after collision with the table.

Although the third law of friction states the coefficient of friction is constant regardless of the sliding velocity, this is generally not valid in the real world (Braun and Peyrard, 2011). This implies that the coefficient of friction can be influenced by initial velocities of the contact point of the ball with the table. Therefore, the velocity of the contact point of the ball with the table ( $v_{\text {cp_before }}$ ) was calculated using the following equation:

$$
v_{c p \_b e f o r e}=v_{h_{-} \text {before }}+r * \omega_{\text {before }}
$$

where $r$ is the radius of the ball.

Prediction of velocity and trajectory after collision

The coefficients of restitution for a given $v_{z_{-} b e f o r e}$ and coefficients of friction for a given

$$
v_{\text {cp_before }} \text { were predicted via linear }
$$

regression. Based on the predicted coefficients of restitution and friction, the velocities of the balls after collision for a given initial condition were predicted. Then, the trajectories of the ball after collision with the table were predicted for the assumed service, smash and top-spin drive by time-integrating the equation of motion of the ball defined by Tanaka et al. (2014) using the fourth Runge Kutta algorithm. The equations of motion were as follows:

$$
\begin{gathered}
\frac{d x}{d t}=v_{h} \\
\frac{d z}{d t}=v_{z} \\
\frac{d v_{h}}{d t}=-\frac{1}{2} D v_{h} \sqrt{v_{h}^{2}+v_{z}^{2}}-\frac{1}{2} L v_{z} \sqrt{v_{h}{ }^{2}+v_{z}^{2}} \\
\frac{d v_{z}}{d t}=-g-\frac{1}{2} D v_{z} \sqrt{v_{h}^{2}+v_{z}^{2}} \\
+\frac{1}{2} L v_{h} \sqrt{v_{h}^{2}+v_{z}^{2}} \\
D=C_{D} \rho \pi r^{2} / m \\
L=C_{L z} \rho \pi r^{2} / m
\end{gathered}
$$

where $x$ and $z$ are the horizontal and vertical positions of the center of the ball, $v_{h}$ and $v_{z}$ are the horizontal and vertical components of velocities of the center of the ball, $C_{D}$ is the drag coefficient, $C_{L Z}$ is the lift coefficient, $\rho$ is the air density $\left(1.293 \mathrm{~kg} / \mathrm{m}^{3}\right)$, and $m$ is the mass of the ball. The drag coefficient and lift coefficient were assumed to be constant between the two types of balls. Based on a previous report by Tanaka et al. (2014), the drag coefficient $\left(C_{D}\right)$ was set to 0.480 for back-spin and 0.495 for top-spin, and the lift coefficient $\left(C_{L Z}\right)$ was set to 0.200 for back-spin and - 0.288 for top-spin. The trajectories of the balls were predicted for duration of $0.5 \mathrm{~s}$ for a service and $0.1 \mathrm{~s}$ for a smash and a drive.

\section{Statistics}

Simple regression analysis was conducted to compute the regression coefficient and the regression equation between the $v_{z_{-}}$before and the coefficient of restitution, and the $v_{c p \_b e f o r e}$ and the coefficient of friction. Analysis of Covariance (ANCOVA) was used to compare the regression lines between celluloid and plastic balls. The main factor was the difference in ball material (celluloid or plastic) and the covariate was $v_{z_{-} \text {before }}$ for the coefficient of restitution and $v_{c p \_b e f o r e}$ for the coefficient of friction. The existence of significant interaction between the main factor and the covariate implied that the 
regression lines were not parallel and the regression coefficients were different (the level of significance set at $p<0.01$ ).

\section{Results}

The coefficient of restitution was similar between the two types of the ball when the $v_{z_{-} b e f o r e}$ initial conditions were slower. However, the coefficient of restitution was higher in plastic balls than in celluloid ones when $v_{z_{-} \text {before }}$ initial conditions were relatively higher (Figure 1a). A significant correlation between $v_{z_{-}}$before and the coefficient of restitution was found for both types of balls $(r=0.97, p<0.01$ for celluloid balls and $r=0.96, p<0.01$ for plastic balls). The ANCOVA results showed the presence of a significant effect due to the difference in ball material $(p<0.01, \mathrm{~F}$-value $=59.835)$. Furthermore, there was a significant interaction effect between the difference in ball material and $v_{z_{-} \text {before }}$ on the coefficient of restitution $(p<0.01$, F-value $=$ 1835.939), which means there was a significant difference between the regression coefficients of the two types of balls (regression line equations: $y$ $=-5.8^{*} 10^{-3}+1.002$ for celluloid balls and $\mathrm{y}=$ $-9.6^{*} 10^{-3}+1.0418$ for plastic balls).

The coefficient of friction was also higher for plastic than celluloid balls when $v_{c p \_b e f o r e}$ values were relatively smaller; however, the difference between the two types of the ball was smaller when $v_{c p \_b e f o r e}$ values were higher (Figure 1b). A significant correlation between $v_{\text {cp_before }}$ and the coefficient of friction was found for both types of balls $(r=0.96, p<0.01$ for celluloid balls and $r=0.92, p<0.01$ for plastic balls). The ANCOVA results showed the presence of a significant effect due to the difference in ball material $(p<0.01, \mathrm{~F}$-value $=317.325)$. In addition, there was a significant interaction effect between the difference in ball material and $v_{c p \_b e f o r e}$ on the coefficient of friction $(p<0.01$, F-value $=$ 1202.908), which means that there was significant difference between the regression coefficients of the two types of balls (regression line equations: $y$ $=0.0017 \mathrm{x}+0.1635$ for celluloid balls and $\mathrm{y}=$ $0.0011 x+0.2526$ for plastic balls). The horizontal deceleration rates $\left(\frac{\Delta v_{h}}{v_{h-\text { before }}}\right)$ were greater for plastic balls than for celluloid ones when compared at the same value of $v_{\text {cp_before }}$ (Figure 2).
The post-collision trajectories of balls of a different type having the same initial conditions were compared. To simulate a service, the initial conditions were set as follows: $v_{h \_ \text {before }}=21.0$ $\mathrm{km} / \mathrm{h}, v_{z_{-} \text {before }}=-8.0 \mathrm{~km} / \mathrm{h}$, and spin rate $=60$ rps (backspin) according to Yoshida (2014). The computed conditions of the ball after collision for celluloid and plastic balls were as follows: $v_{h_{-} \text {after }}=17.2 \mathrm{~km} / \mathrm{h}, v_{z_{-} \text {after }}=7.7 \mathrm{~km} / \mathrm{h}$, and spin rate $=47 \mathrm{rps}$ (backspin) for the celluloid ball, and $v_{h \_a f t e r}=16.3 \mathrm{~km} / \mathrm{h}, v_{z_{-} a f t e r}=7.7 \mathrm{~km} / \mathrm{h}$, and spin rate $=44 \mathrm{rps}$ (backspin) for the plastic ball. Because of the greater deceleration in plastic balls during collision, the simulated trajectories for 0.5 $\mathrm{s}$ were different between ball types (Figure 3a). At $0.5 \mathrm{~s}$, the plastic ball was located $9.3 \mathrm{~cm}$ behind and $1.9 \mathrm{~cm}$ below the position of the celluloid ball at the same instant.

To simulate a smash, the initial conditions were set as follows: $v_{h_{-} \text {before }}=77 \mathrm{~km} / \mathrm{h}$, $v_{z_{-} \text {before }}=-28 \mathrm{~km} / \mathrm{h}$, and spin rate $=60 \mathrm{rps}$ (topspin). The computed conditions of the ball after collision for celluloid and plastic balls were as follows: $v_{h_{-} \text {after }}=64.8 \mathrm{~km} / \mathrm{h}$ and $v_{z_{-} \text {after }}=$ $21.7 \mathrm{~km} / \mathrm{h}$ for the celluloid ball, and $v_{h_{-}}$after $=$ $61.2 \mathrm{~km} / \mathrm{h}$ and $v_{z_{-} \text {after }}=23.5 \mathrm{~km} / \mathrm{h}$ for the plastic ball. Because of the greater horizontal deceleration and higher bounce of the plastic ball during collision, the simulated trajectories for $0.1 \mathrm{~s}$ were different between both types of the ball (Figure 3b). At $0.1 \mathrm{~s}$, the plastic ball was located $7.7 \mathrm{~cm}$ behind and $5.7 \mathrm{~cm}$ above the position of the celluloid ball at the same instant.

To simulate a drive (a fast ball with greater topspin is called "drive" in table tennis) trajectory, two sets of initial conditions with the same speed but different spin rates were set as follows: $v_{h_{-} \text {before }}=42 \mathrm{~km} / \mathrm{h}, v_{z_{-} \text {before }}=-24 \mathrm{~km} / \mathrm{h}$, and spin rate $=100 \mathrm{rps}$ and $170 \mathrm{rps}$ (topspin). With a spin rate of $100 \mathrm{rps}$, the computed post-collision conditions were as follows: $v_{h \_a f t e r}=43.3$ $\mathrm{km} /$ hand $v_{z_{-} \text {after }}=19.5 \mathrm{~km} / \mathrm{h}$ for the celluloid ball, and $v_{h_{-} a f t e r}=43.4 \mathrm{~km} / \mathrm{h}$ and and $v_{z_{-} a f t e r}=$ $20.7 \mathrm{~km} / \mathrm{h}$ for the plastic ball. As a result, the difference in the simulated trajectories under this condition for $0.1 \mathrm{~s}$ was small compared to the other conditions (the plastic ball was located 2.9 $\mathrm{cm}$ behind and $0.4 \mathrm{~cm}$ below the celluloid ball (Figure 3c)). With a spin rate of $170 \mathrm{rps}$, the 
computed post-collision conditions were as follows: $v_{h_{-} \text {after }}=46.8 \mathrm{~km} / \mathrm{h}$ and $v_{z_{-} \text {after }}=19.5$ $\mathrm{km} / \mathrm{h}$ for the celluloid ball, and $v_{h \_}$after $=51.9$ $\mathrm{km} / \mathrm{h}$ and $v_{Z_{-} \text {after }}=20.7 \mathrm{~km} / \mathrm{h}$ for the plastic ball. Because of the greater horizontal acceleration and higher bounce of the plastic ball during collision, the simulated trajectories for 0.1 $\mathrm{s}$ were different between the balls (Figure 3d). At $0.1 \mathrm{~s}$, the plastic ball was $11.8 \mathrm{~cm}$ ahead of and 1.3 $\mathrm{cm}$ above the position of the celluloid ball at the same instant.

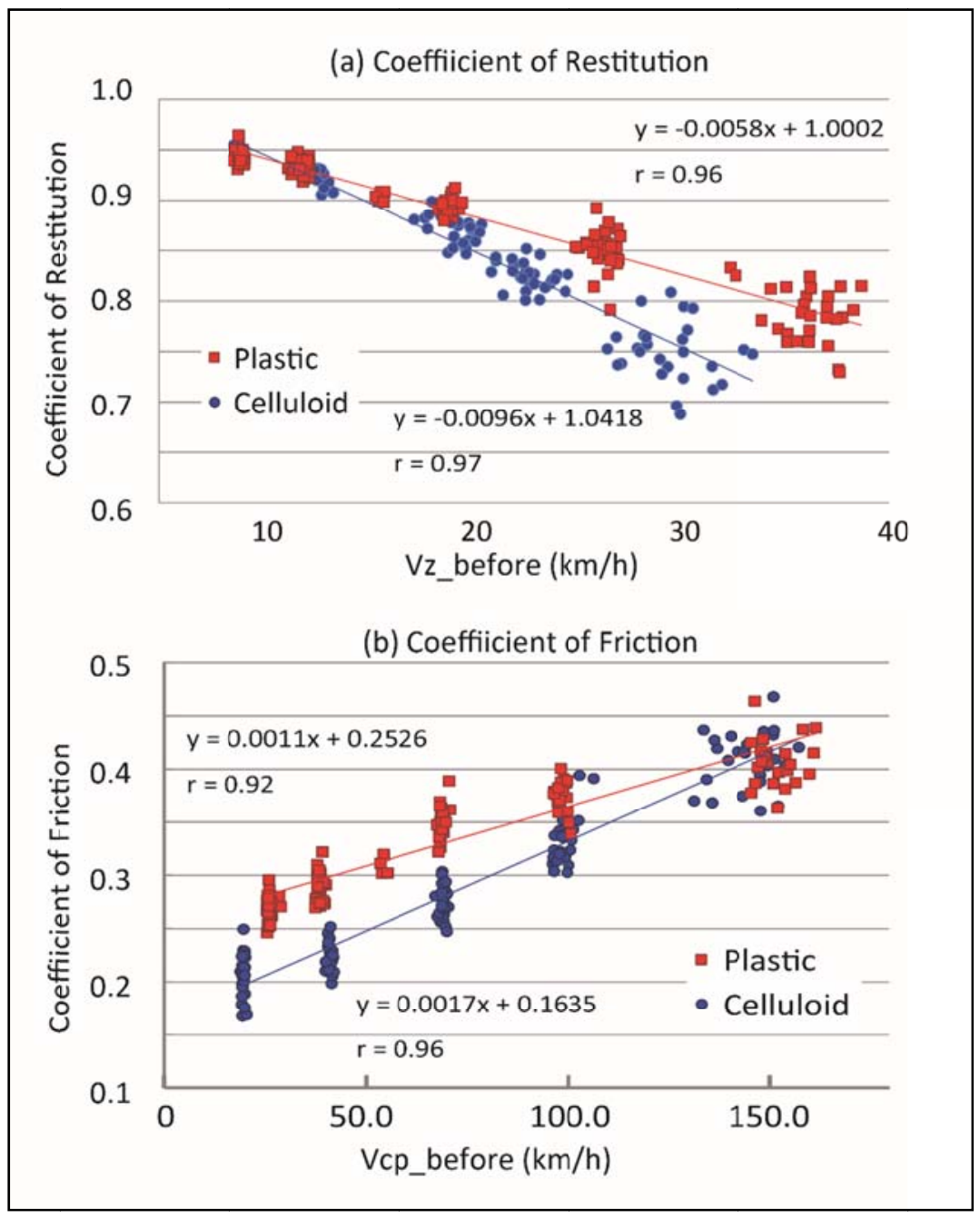

Figure 1

Coefficients of restitution and friction for celluloid balls and plastic balls. (a) Coefficients of restitution plotted against initial vertical velocity of the ball center ( $\mathbf{V}_{\mathbf{z} \text { before }}$ ).

(b) Coefficients of friction for celluloid balls and plastic balls plotted against initial contact point velocity of the ball ( $\left.\mathbf{v}_{\mathbf{c p} \_ \text {before }}\right)$. 


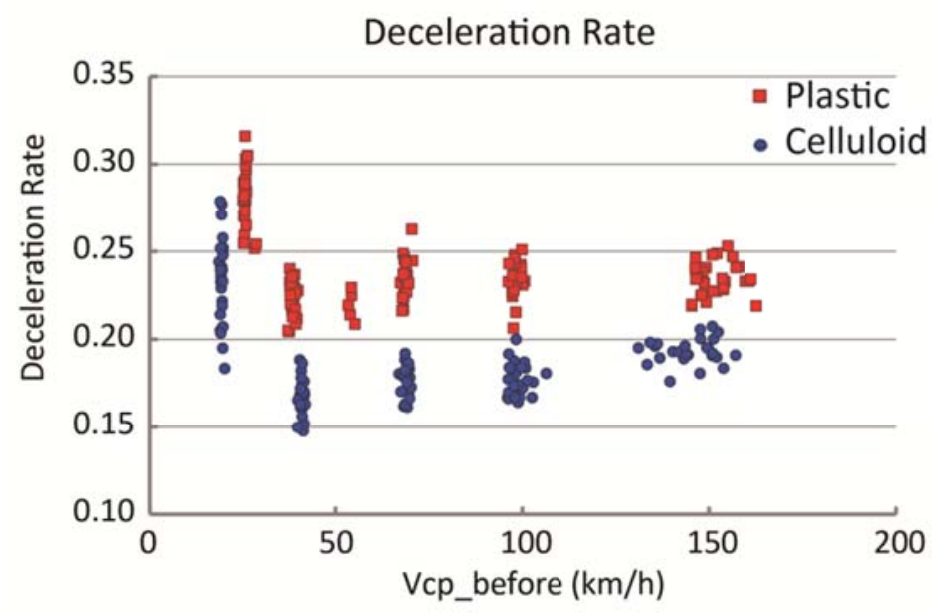

Figure 2

Deceleration rate of the horizontal velocities of the center of the ball $\left(\mathbf{V}_{\mathbf{h} \_ \text {before }}\right)$ for celluloid balls and plastic balls plotted against initial contact point velocity of the

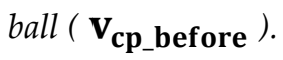

Ball Trajectory Estimation
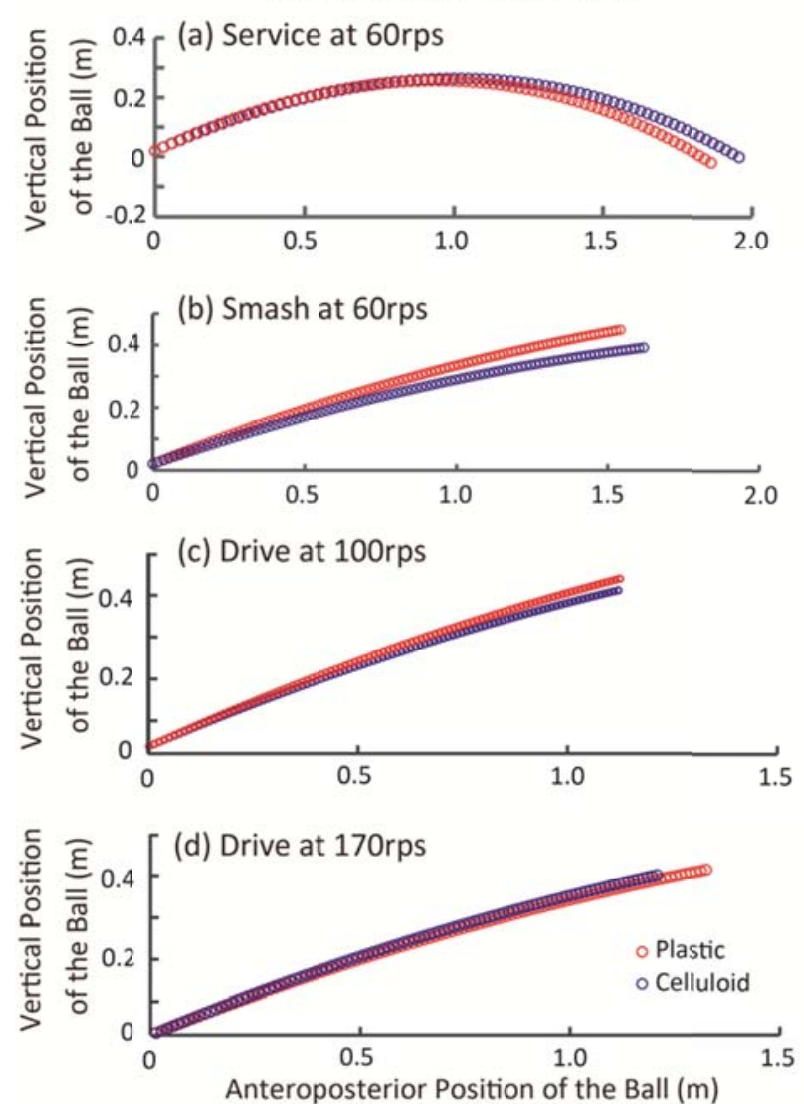

Figure 3

Estimated trajectory of the balls after collision with the table at (a) service conditions,

(b) smash condition, and (c) (d) drive conditions at medium and high top-spin. 


\section{Discussion}

The purpose of this study was to compare the behavior of celluloid balls, which had been long used in table tennis, with that of the newly introduced plastic balls, and understand their differences and similarities. The results of this study show clear differences between the two types of the ball when the initial conditions correspond to the region where differences in the coefficients of restitution and friction are large between the two types of balls. In other words, if the characteristics and behavior of balls are to be investigated, testing at multiple initial conditions should be conducted since the behavior of the balls is greatly influenced by their initial conditions.

The difference in the coefficient of restitution between the two balls was higher when $v_{z_{-}}$before values were relatively higher (Figure 1a). V $\mathrm{z}_{\mathrm{z} \text { after }}$ was determined as the product of the coefficient of restitution $(e)$ and $v_{z_{-} \text {before. }}$ Therefore, greater differences in ball height at the same instant of time were seen for conditions of higher $v_{z_{-}}$before values such as in the case of a smash (Figure $3 b$ ), than in the case of a service (Figure $3 a$ ) or a drive (Figures $3 c$ and $3 d)$. On the other hand, $v_{h_{-} \text {after }}$ was determined by the following equation according to Shibukawa (1969):

$$
v_{h_{-} \text {after }}=v_{h_{-} \text {before }}-\mu(1+e) * v_{z_{-} \text {before }}
$$

This indicates that the horizontal velocity after collision was determined not only by the coefficient of friction, but also by the coefficient of restitution, as well as horizontal and vertical initial velocities of the center of the ball. The horizontal deceleration rates were still different between the two types of the ball for very fast $v_{h_{-} \text {before }}$ values (Figure 2), even though the coefficients of friction were similar at that condition, because the $v_{z_{-} b e f o r e}$ value was higher at the same time, and there were still some differences in $e$ between the two types of the ball. As in the case of a service (Figure 3a), plastic balls experienced greater horizontal deceleration than the celluloid balls. This can be attributed to the lower horizontal velocities and lower horizontal contact point velocity of services, which yields a greater difference in the coefficient of friction between the two ball types (Figure 1b). In case of a smash (Figure $3 b$ ), even though the horizontal contact point velocities were relatively higher compared to the service, there was still a difference between the coefficients of friction of the two types of the ball, and in addition to that, there was a greater difference in their coefficients of restitution. Therefore, for the conditions of a smash, both horizontal and vertical positions of the ball at $0.1 \mathrm{~s}$ were different between the two ball types. Araki et al. (1996) also reported the existence of velocity dependence on normal and tangential coefficients of restitution in the racketball interaction, which is consistent with our data. In addition, when the ball size was changed from 38 to $40 \mathrm{~mm}$ in 2000, similar research investigations were conducted to report the influence of introducing new balls (Iimoto et al., 2002; Takeuchi et al., 2002; Tang et al., 2002). limoto et al. (2002) reported that the difference in the rebound characteristics of the ball was more remarkable for increased ball spin, which also suggests that the degree of influence depends on the initial conditions. Therefore, the degree of the difference between the two types of the ball depends largely on the initial conditions of the ball, whether they correspond to circumstances where the difference in the coefficients of restitution and friction is large or small; thus, testing for multiple initial conditions should be conducted to comprehensively identify differences between two types of balls.

In addition to considering the area associated with greater differences in the coefficients between the two types of the ball, it is necessary to consider the direction of the contact point velocity if differences are expressed in terms such as acceleration and deceleration. With regard to horizontal acceleration and deceleration, since the frictional force depends on the direction of the contact point velocity, the behavior largely depends on the combination of the horizontal velocity of the ball center and the spin rate. In the case of back spin services, the contact point velocity generated by the back spin is oriented towards the same direction that the ball travels. Therefore, the ball experiences a decelerating force from the table during collision. Table tennis players often use the expression that "a ball stops at the collision in services", which describes the deceleration of the horizontal velocity. In services having back-spin, players are expected to encounter more "stops" with plastic balls than 
celluloid ones.

However, services with plastic balls do not always give the impression of more stops than with celluloid balls. If top-spin is applied to the service, then the contact point velocity may be opposite to the direction that the ball travels if $v_{h_{-} b e f o r e}$ is smaller than $r * \omega_{\text {before }}$. In this case, plastic balls have greater potential to accelerate than celluloid balls, which is the opposite phenomenon of "stops" in back-spin services. Although Meyer and Tiefenbacher (2012) reported greater deceleration with plastic balls, the behavior of the ball, with respect to acceleration and deceleration is dependent on the initial conditions. In fact, plastic balls are expected to accelerate more than celluloid balls when greater top-spin is applied, as in the case shown in Figure $3 \mathrm{~d}$, where the direction of the contact point velocity was opposite to the direction in which the ball was traveling, which resulted in an accelerating force applied to the ball by the table. In addition, since the condition matched with the region where the differences in the coefficients of restitution and friction between the two types of the ball were high, the behavioral difference between the two balls was remarkable. In other cases, the contact point velocity approached zero depending on the combination of horizontal velocity of the ball center and the spin rate. As shown in Figure 4c, the difference in the trajectory was smaller in the drive under conditions of $v_{h \_ \text {before }}=42 \mathrm{~km} / \mathrm{h}, v_{z_{-} \text {before }}=-24 \mathrm{~km} / \mathrm{h}$, and spin rate $=100 \mathrm{rps}$. Here, the contact velocity was almost equal to zero, which generated neither acceleration nor deceleration forces upon collision with the table. As a result, not much change occurred after the collision and the behavioral difference was smaller.

To bring all the results together and consider game situations, some adjustments for predicting ball trajectories of plastic balls are suggested. First, as mentioned above, since services with back-spin will experience greater deceleration upon collision with the table, players can serve the ball that does not approach too close the opponent. When the ball decelerates upon collision with the table on the opponent's side and does not go beyond the end of the table, but instead stays within the table boundary, it is more difficult for the receiver to return the ball with great top-spin since the table obstructs the swing of the racket. Therefore, a server can make use of this phenomenon with plastic balls and the receiver should expect a higher possibility of its occurrence with plastic balls than with celluloid ones. In addition, since plastic balls have a higher coefficient of friction with the table, they are expected to present greater changes in trajectories to their post-collision trajectory, not only regarding back-spin, but also side-spin or combinations of back-spin and side-spin. Therefore, players should expect greater changes in trajectories with plastic balls and consider new tactics to make use of these characteristics of plastic balls.

In addition to adjustments in the prediction of ball trajectories of slower balls such as a service, some adjustments are suggested for plays with faster balls such as a smash or a drive. Players should expect drives to accelerate more with a plastic ball having greater top-spin upon collision with the table than a celluloid ball, so the plastic ball approaches the player faster than expected. A smash with a plastic ball having greater initial vertical velocity will bounce off higher than expected in a smash with a celluloid ball. These behavioral changes between the two types of the ball may possibly influence defensive players who play far away from the table (often referred to as a "chopper"). If their opponent smashes a plastic ball, the ball will bounce off higher than expected in a smash with a celluloid ball, giving the player more time to react and hit the ball as precisely as they desire. On the other hand, if the opponent responds to a back-spin ball hit by a chopper with the back-spin "stop" technique, which also decelerates the ball with counter back-spin, causing the plastic ball to experience more stops upon collision with the table, choppers will have to travel back and forth for greater distances. However, since the behavior of the balls upon collision with the racket was not investigated, future research should be conducted if specific tactics are to be recommended with regard to the change in ball materials. Moreover, since only balls having back-spin were tested in this study, further research is required to identify how sidespin or other types of spin with different velocities affects the behavior of the two types of the ball. 


\section{Conclusion}

Changes between the trajectory of celluloid balls, which had been long used in table tennis, and newly introduced plastic balls were investigated in this study. Plastic balls demonstrated a higher coefficient of restitution than celluloid balls when the initial vertical velocities were higher. Moreover, the coefficient of friction was higher for plastic balls when the initial horizontal contact point velocities were lower. As a result, for slower balls with back-spin, as in the case of a service, plastic balls are expected to experience more deceleration upon collision with the table than celluloid balls. On the other hand, for faster balls with greater amounts of top-spin, plastic balls are expected to experience greater acceleration upon collision with the table than celluloid balls. Since the behavior of the ball is largely influenced by the initial conditions, testing at various initial conditions is necessary to understand the characteristics of each type of the ball.

\section{Acknowledgements}

The authors would like to thank the staff at the Japan Table Tennis Association and Japan Institute of Sports Sciences for their support in data collection.

\section{References}

Braun O, Peyrard M. Dependence of kinetic friction on velocity: Master equation approach. Phys Rev E, 2011; 83: 1-9

Iimoto Y, Yoshida K, Yuza N. Rebound characteristics of the new table tennis ball; Differences between the 40mm (2.7 g) and 38mm (2.5 g) balls. Int J Table Tennis Sci, 2002; 5: 233-243

Küneth T. The New Plastic Balls - Questions and Answers, 2015. Available at: http://www.ittf.com/stories/pictures/Plastic_balls_Q_A_v2.pdf; accessed on 21.03.2016

Meyer D, Tiefenbacher K. Comparison of plastic ("poly") and celluloid balls, 2012. Available at: http://etta.co.uk/wp-content/uploads/2013/04/Poly_Celluloid_Balls_Testing.pdf;accessedon 1.03.2016

Ozaki H, Tamaki S, Ikebukuro H, Yamada K, Yoshida K. Development of a table tennis machine to counter the "Chiquita" spin. 31 Int Conf Biomech Sports Conf Proc; 2013

Savitzky A, Golay M. Smoothing and differentiation of data by simplified least squares procedures. Anal Chem, 1964; 36(8): 1627-1639

Shibukawa K. Dynamics of motion. Tokyo: Taishukan-Shoten; 1969

Tamaki S, Saito H. Reconstructing the 3D trajectory of a ball with unsynchronized cameras. Int J Comput Sci Sport, 2015; 14(1): 51-68

Tanaka K, Fukuju T, Miyazaki T, Himeno R. Aerodynamic properties of a table tennis ball. Nagare, 2014; 33: 37-45

Takeuchi T, Kobayashi Y, Hiruta S, Yuza N. The effect of the 40mm diameter ball on table tennis rallies by elite players. Int J Table Tennis Sci, 2002; 5: 278-284

Tang H, Mizoguchi M, Toyoshima S. Speed and spin characteristics of the 40mm table tennis ball. Int J Table Tennis Sci, 2002; 5: 278-284 
Tiefenbacher K, Durey A. The impact of the table tennis ball on the racket. Int J Table Tennis Sci, 1994; 2: 1-14

Yoshida K, Yamada K, Tamaki S, Naito H, Kaga M. The rotation speed of the service ball delivered by world-class table tennis players. Japan Soc Phys Educ Health Sport Sci, 2014; 59(1): 227-236

Xie W, Teh KC, Qin ZF, Speed and spin of 40mm table tennis ball and the effects on elite players. Int Conf Biomech Sports Conf Proc, 2002: 623-626

\section{Corresponding author:}

\section{Yuki Inaba}

Department of Sports Science, Japan Institute of Sports Sciences

3-15-1 Nishigaoka, Kita-ku, Tokyo 115-0056 Japan

Phone: +81-3-5963-0231

Fax: +81-3-5963-0232

E-mail: yuki.inaba@jpnsport.go.jp 